\title{
THE TIME DEPENDENT SPECTRA OF RESONANT SECONDARY EMISSION IN THE CASE OF TWO RELAXATION TIMES
}

INNA REBANE, RESONANTS-SEKUNDAARKIIRGUSE AJAST SOLTUVAD SPEKTRID KAHE RELAKSATSIOONIAJA ARVESTAMISE KORRAL

ИННА РЕБАНЕ. ЗАВИСЯЩИЕ ОТ ВРЕМЕНИ СПЕКТРЫ РЕЗОНАНСНОГО ВТОРИЧНОГО СВЕЧЕНИЯ С УЧЕТОМ ДВУХ ВРЕМЕН РЕЛАКСАЦИИ

\section{(Presented by K. K. Rebane)}

The aim of the present paper is the calculation of time dependent spectra of resonance secondary emission (RSE). Energy and phase relaxations and spectral resolution of the experimental apparatus are taken into account. 1. Introduction. In a previous paper [1] expressions were given describing the time evolution of the RSE spectrum of an impurity centre in crystal by a short pulse of light. The results were illustrated by computer calculations $\left[{ }^{1,2}\right]$. But only one single kind of the relaxation processes was taken into account - the relaxation of the vibrational energy. It means that the results are valid when the processes disturbing the phase memory are absent. For impurity centres it is the case at sufficiently low temperatures of the crystal.

Here we take into account the processes leading to phase (transvense) relaxation without energy loss. The relaxation processes with vibrational energy loss we characterize by the longitudinal relaxation time $\tau_{\|}$and the transverse one by relaxation time $\tau_{\perp}$. The influence of the experimental apparatus is characterized by its spectral resolution $\eta$ and taken into account as well.

Two kinds of relaxation are particularly important for the classification of the RSE into resonant scattering (RS), ordinary luminescence and hot luminescence (HL); the $\tau_{\perp}$ being the crucial parameter for distinguishıng between RS and HL $\left.{ }^{3}\right]$.

The centre is supposed to have two electronic states and one local vibration, described in Born-Oppenheimer adiabatic approximation by two potential curves, having different equilibrium positions and vibrational frequencies $\omega_{1}$ and $\omega_{2}$. The local vibration interacts with the thermal bath (lattice phonons) and is involved in energy $\left(\tau_{\|}\right)$and phase $\left(\tau_{\perp}\right)$ relaxation processes.

2. The Recorded RSE Spectra. Let $\Omega$ be the RSE frequency. The recorded spectrum of the RSE is calculated by the formula [ $\left.{ }^{4}\right]$

$$
\begin{gathered}
I(\Omega, t)=2 \operatorname{Re} \iint_{0}^{\infty} \int d \mu d \nu d \tau d \tau^{\prime} \mathrm{e}^{i \Omega \mu} S\left(t-\mu-v-\tau^{\prime}, t-v-\tau\right) \times \\
\times C(\mu+v, v) a\left(\mu, \tau, \tau^{\prime}\right),
\end{gathered}
$$


where $S\left(t_{1}, t_{2}\right)=\exp \left[i_{\omega_{0}}\left(t_{1}-t_{2}\right)-\Delta\left|t_{1}\right|-\Delta\left|t_{2}\right|\right]$ is the correlation function of the excitation field $(\Delta-$ the half-width of the exciting pulse $\left(\Delta \ll \omega_{1}, \omega_{2}\right), \omega_{0}$ - the frequency at the spectral maximum of the excitation pulse); $C\left(t_{1}, t_{2}\right)=\exp \left[-\eta\left|t_{1}\right|-\eta\left|t_{2}\right|\right]$ is the recording correlation function; the correlation function of impurity centre $a\left(\mu, \tau, \tau^{\prime}\right)$ is taken from $\left[{ }^{3}\right]$ and multiplied by the term which describes phase relaxation $\left[{ }^{5}\right]$ :

$$
\begin{gathered}
\exp \left[-\Gamma_{\perp}|\mu|-\Gamma_{\perp}\left|\mu+\tau^{\prime}-\tau\right|-\Gamma_{\perp} \tau-\Gamma_{\perp} \tau^{\prime}+\Gamma_{\perp}\left|\mu+\tau^{\prime}\right|+\Gamma_{\perp}|\mu-\tau|\right], \\
\left(\Gamma_{\perp}=\left(1 / 2 \tau_{\perp}\right)^{-1}\right) .
\end{gathered}
$$

Let $M$ be the quantum number of the vibrational level of the excited electronic state into which the centre is excited; $L_{2}$ and $L_{1}$ - the numbers of the initial and final vibrational states of the radiational vibronic transition.

The recorded spectrum can be presented as a sum, each member of which gives the intensity of a spectral line corresponding to a transition between two fixed vibronic levels:

$$
I(\Omega, t)=\sum_{L_{1}=0}^{\infty} \sum_{L_{2}=0}^{M} I L_{2} L_{1}(\Omega, t),
$$

where (at $t \geqslant 0)$

$$
\begin{gathered}
I_{2}^{L_{2} L_{1}}(\Omega, t) \simeq|\langle 0 \mid M\rangle|^{2} \cdot\left|\left\langle L_{2} \mid L_{1}\right\rangle\right|^{2}\left(\begin{array}{l}
M \\
L_{2}
\end{array}\right) \sum_{k=0}^{M-L_{2}}\left(\begin{array}{c}
M-L_{2} \\
k
\end{array}\right)(-1)^{M-L_{2}+k} \cdot \mathrm{e}^{-2 \eta t} . \\
{\left[\sum_{i=1}^{6} \frac{C_{i, h}^{L_{2} L_{1}}(\Omega)}{\alpha_{i, h}^{L_{2} L_{1}}(\Omega)}\left(1-\exp \left(\alpha_{i, h}^{L_{2} L_{1}}(\Omega) t\right)\right)+\frac{C_{7, h}^{L_{2} L_{1}}(\Omega)}{2 \Delta+2 \eta}+c . c .\right] .}
\end{gathered}
$$

Here $|\langle 0 \mid M\rangle|^{2}$ and $\left|\left\langle L_{2} \mid L_{1}\right\rangle\right|^{2}$ are the Franck-Condon factors for absorption $0 \rightarrow M$ and emission $L_{2} \rightarrow L_{1}$;

$$
\begin{aligned}
& \alpha_{1, k}^{L_{2} L_{1}}(\Omega)=i \Delta \Omega_{L_{2} L_{1}}-\Gamma_{\|}\left(L_{1}+L_{2}\right)-\gamma / 2-\Gamma_{\perp}+\eta, \\
& \alpha_{2, k}^{L_{2} L_{1}}(\Omega)=i\left(\Delta \Omega_{M L_{1}}+x\right)-\Gamma_{\|} L_{1}-\Delta+\eta, \\
& \alpha_{3, h}^{L_{2} L_{1}}(\Omega)=-2 \Gamma_{\|}(M-k)-\gamma+2 \eta, \\
& \alpha_{4, k}^{L_{2} L_{1}}(\Omega)=i x-\Gamma_{\|} M-\gamma / 2-\Gamma_{\perp}-\Delta+2 \eta, \\
& \alpha_{5, h}^{L_{2} L_{1}}(\Omega)=\left[\alpha_{4, k}^{L_{2} L_{1}}(\Omega)\right]^{*}, \\
& \alpha_{6, k}^{L_{2} L_{1}}(\Omega)=-2 \Delta+2 \eta .
\end{aligned}
$$

The factors $C_{i, k}^{L_{2} L_{1}}(\Omega)$ are different for $L_{2}<M$ and $L_{2}=M$ :

$$
\begin{aligned}
& C_{1, k}^{L_{2} L_{1}}(\Omega)=A_{3}\left[i \Delta \Omega_{L_{2} L_{1}}-\Gamma_{\|}\left(L_{1}+L_{2}\right)-\gamma / 2-\Gamma_{\perp}-2 \Delta-\eta\right]^{-1}- \\
& -C_{3, k}^{L_{2} L_{1}}(\Omega)-C_{4, k}^{L_{2} L_{4}}(\Omega)-C_{5_{2}, k}^{L_{2} L_{4}}(\Omega)-C_{6, k}^{L_{2} L_{2}}(\Omega) ; \\
& C_{2, \ell}^{L_{2} L_{1}}(\Omega)=0 \text {; } \\
& C_{3, k}^{L_{2} L_{1}}(\Omega)=\left(-A_{2}+A_{3}-A_{4}-A_{4}^{*}\right)\left[i \Delta \Omega_{L_{2} L_{1}}+\Gamma_{\|}\left(2(M-k)-L_{1}-L_{2}\right)+\right. \\
& \left.+\gamma / 2-\Gamma_{\perp}-\eta\right]^{-1}
\end{aligned}
$$




$$
\begin{aligned}
& C_{4, k}^{L_{2} L_{i}}(\Omega)=A_{4}^{*}\left[i\left(\Delta \Omega_{L_{2} L_{4}}-x\right)-\Gamma_{\|}\left(L_{1}+L-M\right)+\Delta-\eta\right]^{-1} ; \\
& C_{5, h}^{L_{2} L_{1}}(\Omega)=A_{4}\left[i\left(\Delta \Omega_{L_{2} L_{1}}+x\right)-\Gamma_{\|}\left(L_{1}+L_{2}-M\right)--\Delta-\eta\right]^{-1} \text {; } \\
& C_{6, h}^{L_{2} L_{1}}(\Omega)=A_{2}\left[i \Delta \Omega_{L_{2} L_{1}}-\Gamma_{\|}\left(L_{1}+L_{2}\right)-\gamma / 2-\Gamma_{\perp}+2 \Delta-\eta\right]^{-1} \text {; } \\
& C_{7^{2}, k^{2}}^{L_{1} L_{1}}(\Omega)=-A_{3}\left[i \Delta \Omega_{L_{2} L_{1}}-\Gamma_{\|}\left(L_{1}+L_{2}\right)-\gamma / 2-\Gamma_{\perp}-2 \Delta-\eta\right]^{-1} ; \\
& C_{3,0}^{M L_{1}}(\Omega)=\left[C_{3,0}^{L_{3} L_{1}}(\Omega)\right]_{L_{2}=M} ; \quad C_{5,0}^{M L_{1}}(\Omega)=\left[C_{5,0}^{L_{1} L_{1}}(\Omega)\right]_{L_{2}=M} ; \\
& C_{1,0}^{M L_{1}}(\Omega)=-C_{3,0}^{M L_{1}}(\Omega)+\left(A_{1}-A_{2}\right)\left[i \Delta \Omega_{M L_{1}}-\Gamma_{\|}\left(M+L_{1}\right)-\right. \\
& \left.-\gamma / 2-\Gamma_{\perp}+2 \Delta-\eta\right]^{-1}+\left(A_{5}-A_{1}-A_{4}^{*}\right)\left[i\left(\Delta \Omega_{M L_{1}}-x\right)-\right. \\
& \left.-\Gamma_{\|} L_{1}+\Delta-\eta\right]^{-1}+\left(A_{3}-A_{6}\right)\left[i \Delta \Omega_{M L_{1}}-\Gamma_{\|}\left(M+L_{1}\right)-\right. \\
& \left.-\gamma / 2-\Gamma_{\perp}-2 \Delta-\eta\right]^{-1}-C_{5,0}^{M L_{1}}(\Omega)+ \\
& +\left(A_{6}-A_{5}\right)\left[i\left(\Delta \Omega_{M L_{1}}+x\right)-\Gamma_{\|} L_{1}-\Delta-\eta\right]^{-1} ; \\
& C_{2,0}^{M L_{1}}(\Omega)=\left(A_{1}-A_{5}\right)\left[i \Delta \Omega_{M L_{1}}+\Gamma_{\|}\left(M-L_{1}\right)+\gamma / 2+\Gamma_{\perp}-\eta\right]^{-1}- \\
& -A_{1}\left[i\left(\Delta \Omega_{M L_{1}}+x\right)-\Gamma_{\|} L_{1}+\Delta-\eta\right]^{-1}+ \\
& +A_{5}\left[i\left(\Delta \Omega_{M L_{1}}+x\right)-\Gamma_{\|} L_{1}-\Delta-\eta\right]^{-1} \text {; } \\
& \begin{aligned}
C_{4,0}^{M L_{1}}(\Omega) & =\left(A_{5}-A_{1}\right)\left[i \Delta \Omega_{M L_{1}}+\Gamma_{\|}\left(M-L_{1}\right)+\gamma / 2+\Gamma_{\perp}-\eta\right]^{-1}+ \\
& +\left(A_{1}+A_{4}^{*}-A_{5}\right)\left[i\left(\Delta \Omega_{M L_{1}}-x\right)-\Gamma_{\|} L_{1}+\Delta-\eta\right]^{-1}
\end{aligned} \\
& C_{6,0}^{M L_{1}}(\Omega)=A_{1}\left[i\left(\Delta \Omega_{M L_{1}}+x\right)-\Gamma_{\|} L_{1}+\Delta-\eta\right]^{-1}+ \\
& +\left(A_{2}-A_{1}\right)\left[i \Delta \Omega_{M L_{1}}-\Gamma_{\|}\left(M+L_{1}\right)-\gamma / 2-\Gamma_{\perp}+2 \Delta-\eta\right]^{-1} ; \\
& C_{7,0^{i}}^{M L_{i}}(\Omega)=\left(A_{6}-A_{3}\right)\left[i \Delta \Omega_{M L_{1}}-\Gamma_{\|}\left(M+L_{1}\right)-\gamma / 2-\Gamma_{\perp}-2 \Delta-\eta\right]^{-1}- \\
& -A_{6}\left[i\left(\Delta \Omega_{M L_{1}}+x\right)-\Gamma_{\|} L_{1}-\Delta-\eta\right]^{-1} .
\end{aligned}
$$

The factors $A_{i}$ depend only on the absorption conditions:

$$
\begin{aligned}
A_{1} & =\left[x^{2}+\left(\Gamma_{\|} M+\gamma / 2+\Gamma_{\perp}-\Delta\right)^{2}\right]^{-1} ; \\
A_{2} & =2\left(\Gamma_{\|} M+\gamma / 2+\Gamma_{\perp}-\Delta\right)\left[( 2 \Gamma _ { \| } ( M - k ) + \gamma - 2 \Delta ) \left(x^{2}+\left(\Gamma_{\|} M+\gamma / 2+\right.\right.\right. \\
& \left.\left.\left.+\Gamma_{\perp}-\Delta\right)^{2}\right)\right]^{-1} ; \\
A_{3} & =2\left(\Gamma_{\|} M+\gamma / 2+\Gamma_{\perp}+\Delta\right)\left[( 2 \Gamma _ { \| } ( M - k ) + \gamma + 2 \Delta ) \left(x^{2}+\left(\Gamma_{\|} M+\gamma / 2+\right.\right.\right. \\
& \left.\left.\left.+\Gamma_{\perp}+\Delta\right)^{2}\right)\right]^{-1} ; \\
A_{4} & =2 \Delta\left[( i x - \Gamma _ { \| } ( M - 2 k ) - \gamma / 2 + \Gamma _ { \perp } + \Delta ) \left(\left(i x+\Gamma_{\|} M+\gamma / 2+\Gamma_{\perp}\right)^{2}-\right.\right. \\
& \left.\left.\quad-\Delta^{2}\right)\right]^{-1} ; \\
A_{5}= & -\left[\left(i x+\Gamma_{\|} M+\gamma / 2+\Gamma_{\perp}-\Delta\right)\left(i x-\Gamma_{\|} M-\gamma / 2-\Gamma_{\perp}-\Delta\right)\right]^{-1} ; \\
A_{6}= & {\left[x^{2}+\left(\Gamma_{\|} M+\gamma / 2+\Gamma_{\perp}+\Delta\right)^{2}\right]^{-1} . }
\end{aligned}
$$


Here $\Delta \Omega_{L_{2} L_{1}} \equiv \Omega-\Omega_{21}+\omega_{1} L_{1}-\omega_{2} L_{2} ; x \equiv \Omega_{21}-\omega_{0}+\omega_{2} M$, where $\Omega_{21}$ is the pure electronic energy; $\gamma$ - the radiation width of the excited electronic state. As in [3,1] the relaxation time of the energy of the $n$-th vibrational level $\tau_{\|, n}$ is supposed to be $\tau_{\|, n}=\left(2 n \Gamma_{\|}\right)^{-1}$, where $\Gamma_{\|}$is the energy relaxation half-width of the level $n=1$.

3. Discussion.

1. If we put $\Gamma_{\perp}=0$ and $\eta=0$, the equations (1)-(6) turn into the corresponding equations of $\left[{ }^{1}\right]$.

2. As it was to be expected, the general properties of the time behaviour of the RSE given by the equations above are in good accordance with the results obtained for the one relaxation time case in $\left.{ }^{1,2}\right]$.

3. The most reasonable way to explain and discuss the results (1) (6) is to display them via computer calculations. Only slight elaborations of the computer programs used in $\left[{ }^{1,2}\right]$ are needed. Calculations are in progress.

\section{REFERENCES}

1. Rebane, I. On the theory of time dependence of resonant secondary emission spectra of impurity centres. - ENSV TA Toimet., Füüs. Matem., 1978, v. 27, N 2, p. $192-201$.

2. Реб ане И., Туул А. Контур чисто электропной линии в зависящих от времени спектрах: сужение и осцилляции. - Изв. АН ЭССР, Физ. Матем., 1978, т. 27 , № 4 , с. $463-465$.

3. Hizhnyakov, V., Tehver, I. On the theory of hot luminescence and resonant Raman effect of impurity centres. - Phys. stat. sol., 1970, v. 39, p. 67-78.

4. Хи жняков В. В., Р еб ан е И. К. Зависящие от времени спектры резонансного вторичного свечения. - ЖЭТФ. 1978, т. 74 , вып. 3, с. $885-896$.

5. Hizhnyakov, V., Tehver, I. Theory of resonant secondary, radiation due to impurity centres in crystals. - Phys. stat. sol., 1967, v. 21, p. 755-768.

Academy of Sciences of the Estonian SSR, Institute of Physics

Received

August 21, 1978

After rectification

September 18,1978 